\title{
The Clinical Significance of Seminoma Component in Testicular Mixed Germ Cell Tumour
}

\author{
Serkan Akan ${ }^{a}$ Caner Ediz $^{a}$ Hasan Hüseyin Tavukcu ${ }^{a}$ Alpaslan Ozgun ${ }^{b}$ \\ Omer Yilmaz ${ }^{\mathrm{a}}$ \\ ${ }^{a}$ Department of Urology, University of Health Sciences, Sultan Abdulhamid Han Training and Research Hospital, \\ Istanbul, Turkey; ${ }^{b}$ Department of Medical Oncology, University of Health Sciences, Sultan Abdulhamid Han Training \\ and Research Hospital, Istanbul, Turkey
}

\section{Keywords}

Testicular cancer - Mixed germ cell tumour - Seminoma ·

Prognosis - Occult metastatic disease

\begin{abstract}
Background: The aim of this study was to examine clinical/ pathological characteristics, prognosis and tendency to metastasis of mixed germ cell tumours (MGCTs) that contain a seminoma component. Methods: A total of 111 MGCT cases between 2008 and 2018 were retrospectively enrolled. The patients were divided into 2 groups according to the absence (group 1) or presence (group 2) of seminoma component in MGCTs. Patients' age, complaints at admission to our clinic, primary tumour localization, primary tumour size, preoperative testicular tumour markers, MGCT histopathological components and percentages, lymphovascular invasion, pathological tumour stage, postoperative testicular tumour markers, presence of lymph node involvement in abdominal tomography, lung metastasis based on thorax tomography, clinical tumour stage, adjunctive therapies performed, state of recurrence and survival were compared in 2 groups. $\boldsymbol{R e}$ sults: The mean age of the patients was $24.51 \pm 4.79$ years. The mean age, initial complaint rates, primary tumour size,
\end{abstract}

postoperative testicular tumour markers, presence of lymphovascular invasion, presence of lymph node involvement and lung metastasis were found to be higher in group 2 than in group 1, although these differences were not statistically significant. Especially, it was found that a seminoma component rate of $30 \%$ and higher had a higher tendency for a poor prognosis. Conclusion: Although the word "seminoma" may be initially interpreted as an indication of good prognosis, a seminoma component in MGCTs is actually not a good prognostic factor. MGCTs that contain a seminoma component (especially $30 \%$ and higher) can have a higher tendency for occult metastatic disease.

(c) 2020 S. Karger AG, Basel

\section{Introduction}

Testicular cancer accounts for nearly $1 \%$ of the tumours identified in men. It is the most commonly encountered solid malignancy in men between the ages of 15 and 35 years [1]. Primary testicular tumours can originate from germ cells, sex cord cells or, less commonly, from peritubular stromal and haematopoietic cells [2]. More than $90 \%$ of testicular cancers are malignant and

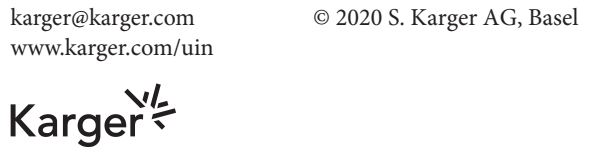

Dr Serkan Akan

Department of Urology, University of Health Sciences Sultan Abdulhamid Han Research and Training Hospital Selimiye Mh., Tibbiye Cd., TR-34668 Istanbul (Turkey) drserkanakan@hotmail.com 
originate from the germ cells. These germ cell tumours are divided into 2 main groups: seminomas and nonseminoma germ cell tumours (NSGCTs). NSGCTs represent various groups of neoplasms, including embryonal carcinomas, yolk sac tumours, choriocarcinomas, teratomas as well as mixed tumours containing the aforementioned types of tumours at varying degrees.

Mixed germ cell tumours (MGCTs) contain multiple non-seminoma components. Cases that show seminoma content along with non-seminoma components are still classified as MGCT, even if the seminoma is the main component [3]. Pure seminomas have usually excellent prognosis, in rare cases the prognosis may be poor. However, there is limited information in the literature regarding the impact of the presence of a seminoma component on MGCT prognosis.

In the present study, a 10-year series was retrospectively scanned to examine the clinicopathological characteristics, prognosis and tendency to metastasise of all MGCTs and those having a seminoma component.

\section{Materials and Methods}

A total of 221 testicular cancer cases that underwent radical inguinal orchiectomy between 2008 and 2018 were retrospectively scanned at our clinic, which was previously a military hospital and is considered as a reference centre for testicular cancer. Of these cases, 143 consisted of NSGCTs. Of the 221 patients, only 111 with fully accessible data were included in the study. The study was approved by the clinical ethics committee.

The following patient information was examined: age, complaints at admission to our clinic, primary tumour localization, primary tumour size, preoperative testicular tumour markers, MGCT histopathological components and percentages, lymphovascular invasion (LVI), pathological tumour stage, postoperative testicular tumour markers, presence of lymph node involvement in abdominal tomography, lung metastasis based on thorax tomography, clinical tumour stage, adjunctive therapies performed, state of recurrence and survival. The histopathological examination of all patients was performed by a uropathologist experienced in testicular tumours. The patients were examined after being divided into 2 groups, one of which consisted of MGCT cases without a seminoma component (group 1), while the other consisted of MGCT cases with a seminoma component (group 2).

Group 2 was also divided into subgroups according to rate of seminoma components. For the assessment of occult metastatic disease, the cut-off level of the seminoma rate in MGCTs was set at $30 \%$. Thus, homogeneity of patients with MGCT that contain a seminoma component was provided and defined as a cut-off level like in embryonal carcinoma ( $>50 \%)$ that was described in the European Association of Urology guidelines.

The data were analysed using PSPP and Microsoft Excel 2010. The statistical methods used to analyse the study data included descriptive analyses (frequency distributions, percentage, average and standard deviation median); the Kruskal-Wallis $\mathrm{H}$ test and
Mann-Whitney $U$ test to measure the difference between the groups and the $\chi^{2}$ test to reveal the differences between discrete variables. The results were evaluated with a confidence interval of $95 \%$ and according to a significance level of $p<0.05$.

\section{Results}

The total 111 MGCT patients who were included in the study consisted of a group of 68 MGCT patients that lacked a seminoma component (group 1) and another group of 43 MGCT patients that showed the presence of a seminoma component (group 2). The mean follow-up period was 45.06 (min.-max. 6-113) months, and 2 patients passed away during this period because of testicular cancer.

The mean age was $24.51 \pm 4.79(18-41)$ years, and the age distribution was $24.24 \pm 4.63$ years for group 1 and $25.41 \pm 4.97$ years for group 2 . There was no statistically significant difference between the groups in terms of age and the distribution of the tumour localization $(p>0.05)$. The most common complaints of the patients at admission were swollen testicle (31.5\%), palpable mass (30.6\%), pain and swelling (19.8\%) and pain (16.2\%), while the testicular tumour was detected incidentally in $1.8 \%$ of the patients. The difference in the initial complaints between the groups was found to be statistically significant $(p<$ 0.05) (Table 1).

The tumour size measured in the scrotal ultrasound performed before orchiectomy was $39.24 \pm 19.26 \mathrm{~mm}$. The minimum and maximum tumour sizes measured with scrotal ultrasound were 7 and $105 \mathrm{~mm}$, respectively. The difference between the groups was not statistically significant $(p>0.05)$ (Table 1).

Tumour markers ( $\alpha$-fetoprotein, $\beta$-human chorionic gonadotropin and lactate dehydrogenase) on postoperative day 7 were found to be positive in $65(58.6 \%)$ patients and negative in $46(41.4 \%)$ patients. Individual examination of each group revealed that the postoperative positivity for tumour markers was 29 (42.6\%) for group 1 and 17 (39.5\%) for group 2. The difference between the 2 groups was not statistically significant $(p>0.05)$ (Table 2$)$.

The contrast-enhanced tomography of the abdomen performed within the first 30 postoperative days identified a lymph node of $10-20 \mathrm{~mm}$ in $28(25.2 \%)$ patients, a lymph node of $21-50 \mathrm{~mm}$ in $22(19.8 \%)$ patients and a lymph node of $>50 \mathrm{~mm}$ in $8(7.2 \%)$ patients, while 53 (47.7\%) patients did not have any lymph node involvement at all. The difference between the 2 groups was not statistically significant $(p>0.05)$ (Table 2$)$. 
Table 1. Demographics and pathological data of patients with mixed germ cell tumours

\begin{tabular}{|c|c|c|c|c|c|c|c|c|}
\hline & & \multicolumn{2}{|c|}{ Group 1} & \multicolumn{2}{|c|}{ Group 2} & \multicolumn{2}{|c|}{ Total $(n=111)$} & \multirow[t]{2}{*}{$p^{\mathrm{a}}$} \\
\hline & & $n$ & $\%$ & $n$ & $\%$ & $n$ & $\%$ & \\
\hline Age, years & Mean \pm SD & \multicolumn{2}{|c|}{$24.24 \pm 4.63$} & \multicolumn{2}{|c|}{$25.41 \pm 4.97$} & \multicolumn{2}{|c|}{$24.51 \pm 4.79$} & 0.39 \\
\hline \multirow[t]{3}{*}{ Localization } & Right testicle & 39 & 57.3 & 25 & 58.1 & 64 & 57.7 & \multirow[t]{3}{*}{0.52} \\
\hline & Left testicle & 27 & 39.7 & 18 & 41.9 & 45 & 40.5 & \\
\hline & Retro-peritoneal & 2 & 3 & - & - & 2 & 1.8 & \\
\hline \multirow[t]{5}{*}{ Main complaint } & Pain & 13 & 19.1 & 5 & 11.6 & 18 & 16.2 & \multirow[t]{5}{*}{0.015} \\
\hline & Swelling & 28 & 41.2 & 7 & 16.3 & 35 & 31.5 & \\
\hline & Pain and swelling & 9 & 13.2 & 13 & 30.2 & 22 & 19.8 & \\
\hline & Palpable mass & 18 & 26.5 & 16 & 37.2 & 34 & 30.6 & \\
\hline & Incidentally & - & & 2 & 4.7 & 2 & 1.8 & \\
\hline $\begin{array}{l}\text { USG tumour } \\
\text { size, mm }\end{array}$ & Mean \pm SD & \multicolumn{2}{|c|}{$41.37 \pm 21.04$} & \multicolumn{2}{|c|}{$37.6 \pm 16.55$} & \multicolumn{2}{|c|}{$39.24 \pm 19.26$} & 0.45 \\
\hline \multirow[t]{5}{*}{ Histopathology } & $\mathrm{EC}$ & 59 & 86.7 & 34 & 79 & 93 & 83.7 & \\
\hline & $\mathrm{T}$ & 53 & 77.9 & 20 & 46.5 & 73 & 65.7 & \\
\hline & YS & 53 & 77.9 & 17 & 39.5 & 70 & 63 & \\
\hline & $\mathrm{CC}$ & 13 & 19.1 & 4 & 9 & 17 & 15.3 & \\
\hline & $S$ & - & - & 43 & 100 & 43 & 38.7 & \\
\hline
\end{tabular}

USG, ultrasonography; EC, embryonal carcinoma; T, teratoma; YS, yolk sac tumour; CC, choriocarcinoma; S, seminoma. ${ }^{a}$ The results were evaluated at $95 \%$ confidence interval and $p<0.05$ significance level.

Table 2. Comparison of MGCTs according to presence or absence of a seminoma component

\begin{tabular}{|c|c|c|c|c|c|c|}
\hline & & \multicolumn{4}{|c|}{ MGCT } & \multirow[t]{3}{*}{$p$ value } \\
\hline & & \multicolumn{2}{|c|}{ group 1} & \multicolumn{2}{|c|}{ group 2} & \\
\hline & & $n$ & $\%$ & $n$ & $\%$ & \\
\hline \multirow[t]{2}{*}{ Tumour markers ${ }^{b}$} & Negative & 39 & 57.4 & 26 & 60.5 & \multirow[t]{2}{*}{0.74} \\
\hline & Positive & 29 & 42.6 & 17 & 39.5 & \\
\hline \multirow{4}{*}{$\begin{array}{l}\text { Metastasis of } \\
\text { retroperitoneal lymph } \\
\text { node in } \mathrm{CT}^{\mathrm{b}}\end{array}$} & No & 37 & 54.4 & 16 & 37.2 & \multirow[t]{4}{*}{0.23} \\
\hline & $10-20 \mathrm{~mm}$ & 13 & 19.1 & 15 & 34.9 & \\
\hline & $21-50 \mathrm{~mm}$ & 13 & 19.1 & 9 & 20.9 & \\
\hline & $\geq 50 \mathrm{~mm}$ & 5 & 7.4 & 3 & 7.0 & \\
\hline \multirow{2}{*}{ Lung metastasis in $\mathrm{CT}^{\mathrm{b}}$} & Negative & 58 & 85.3 & 34 & 79.1 & \multirow[t]{2}{*}{0.39} \\
\hline & Positive & 10 & 14.7 & 9 & 20.9 & \\
\hline \multirow[t]{2}{*}{ Rate of LVI } & Negative & 42 & 61.8 & 27 & 62.8 & \multirow[t]{2}{*}{0.65} \\
\hline & Positive & 26 & 38.2 & 16 & 37.2 & \\
\hline
\end{tabular}

MGCT, mixed germ cell tumors; CT, computed tomography; LVI, lymphovascular invasion. ${ }^{\text {a }}$ The results were evaluated at $95 \%$ confidence interval and $p<0.05$ significance level. ${ }^{\text {b }}$ Postoperatively.

In the clinical staging performed for all the MGCTs, it was determined that $51(45.9 \%)$ patients were stage I, $39(35.1 \%)$ were stage II and $21(18.9 \%)$ were stage III. In group 1, it was found that $35(51.4 \%)$ patients were stage I, $21(30.8 \%)$ were stage II and $12(17.6 \%)$ were stage III; while in group 2, it was found that 16 (37.2\%) patients were stage I, $18(41.8 \%)$ were stage II and $9(20.9 \%)$ were stage III. The difference between the groups was not statistically significant $(p>0.05)$. A total of 5 patients were identified as having metas- 
Table 3. Comparison of results in group 2 according to the rate of seminoma component

\begin{tabular}{|c|c|c|c|c|c|c|}
\hline & & \multicolumn{4}{|c|}{ Group 2} & \multirow[t]{3}{*}{$p$ value $^{\mathrm{a}}$} \\
\hline & & \multicolumn{2}{|c|}{ seminoma $<30 \%$} & \multicolumn{2}{|c|}{ seminoma $\geq 30 \%$} & \\
\hline & & $n$ & $\%$ & $n$ & $\%$ & \\
\hline Age, years & Mean \pm SD & \multicolumn{2}{|c|}{$26.64 \pm 4.55$} & \multicolumn{2}{|c|}{$23.76 \pm 3.84$} & 0.13 \\
\hline $\begin{array}{l}\text { USG tumour size, } \\
\mathrm{mm}\end{array}$ & Mean \pm SD & \multicolumn{2}{|c|}{$39.96 \pm 18.37$} & \multicolumn{2}{|c|}{$34.04 \pm 16.93$} & 0.2 \\
\hline Main complaint & $\begin{array}{l}\text { Pain } \\
\text { Swelling } \\
\text { Pain and swelling } \\
\text { Palpable mass } \\
\text { Incidentally }\end{array}$ & $\begin{array}{l}4 \\
6 \\
9 \\
7 \\
0\end{array}$ & $\begin{array}{c}15.4 \\
23.1 \\
34.6 \\
26.9 \\
0\end{array}$ & $\begin{array}{l}1 \\
1 \\
4 \\
9 \\
2\end{array}$ & $\begin{array}{r}5.9 \\
5.9 \\
23.5 \\
52.9 \\
11.8\end{array}$ & 0.09 \\
\hline Tumour markers ${ }^{\mathrm{b}}$ & $\begin{array}{l}\text { Negative } \\
\text { Positive }\end{array}$ & $\begin{array}{l}15 \\
11\end{array}$ & $\begin{array}{l}57.7 \\
42.3\end{array}$ & $\begin{array}{r}11 \\
6\end{array}$ & $\begin{array}{l}64.7 \\
35.3\end{array}$ & 0.65 \\
\hline $\begin{array}{l}\text { Metastasis of } \\
\text { retroperitoneal lymph } \\
\text { node in } \mathrm{CT}^{\mathrm{b}}\end{array}$ & $\begin{array}{l}\text { No } \\
10-20 \mathrm{~mm} \\
21-50 \mathrm{~mm} \\
\geq 50 \mathrm{~mm}\end{array}$ & $\begin{array}{r}9 \\
13 \\
3 \\
1\end{array}$ & $\begin{array}{l}34.6 \\
50 \\
11.5 \\
3.8\end{array}$ & $\begin{array}{l}4 \\
5 \\
6 \\
2\end{array}$ & $\begin{array}{l}23.5 \\
29.4 \\
35.3 \\
11.8\end{array}$ & 0.16 \\
\hline $\begin{array}{l}\text { Lung metastasis in } \\
\mathrm{CT}^{\mathrm{b}}\end{array}$ & $\begin{array}{l}\text { Negative } \\
\text { Positive }\end{array}$ & $\begin{array}{r}21 \\
5\end{array}$ & $\begin{array}{l}80.2 \\
19.2\end{array}$ & $\begin{array}{r}13 \\
4\end{array}$ & $\begin{array}{l}76.5 \\
23.5\end{array}$ & 0.73 \\
\hline Rate of LVI & $\begin{array}{l}\text { Negative } \\
\text { Positive }\end{array}$ & $\begin{array}{r}9 \\
17\end{array}$ & $\begin{array}{l}34.6 \\
65.4\end{array}$ & $\begin{array}{r}4 \\
13\end{array}$ & $\begin{array}{l}23.5 \\
76.5\end{array}$ & 0.51 \\
\hline
\end{tabular}

USG, ultrasonography; CT, computed tomography; LVI, lymphovascular invasion. ${ }^{\text {a }}$ The results were evaluated at $95 \%$ confidence interval and $p<0.05$ significance level. ${ }^{b}$ Postoperatively.

tasis in other organs, with 3 patients $(2.7 \%)$ having lung metastasis, 1 patient $(0.9 \%)$ having brain metastasis and 1 patient $(0.9 \%)$ having metastasis in the skeletal system.

MGCTs with a seminoma component were also divided into 2 subgroups, with 1 group including cases that had a histopathological seminoma component rate of $30 \%$ and higher $(n=17)$ and the other group including cases that had a histopathological seminoma component rate of $<30 \%(n=26)$. These 2 groups were then compared in terms of mean age, main initial complaint, size of tumour, postoperative testicular tumour markers, lymph node involvement (based on the abdominal tomography findings), lung metastasis (based on the thorax tomography findings) and LVI rates. The mean age, palpable mass, rate of lymph node involvement, rate of lung metastasis and rate of LVI were found to be higher in patients with a seminoma component rate of $30 \%$ and higher, while the mean size of the tumour and postoperative testicular tumour markers were found to be lower. No statistically significant difference was identified between these 2 groups $(p>0.05)$ (Table 3 ).

In group 1, 15 (22\%) of the 68 patients underwent primary retroperitoneal lymph node dissection (RPLND). Of these 15 patients, 3 (20\%) had live tumour cells, 1 (6.6\%) had teratoma, and $2(13.3 \%)$ had live tumour cells and teratoma simultaneously. In group 1, 19 (27.9\%) of the 68 patients underwent RPLND following chemotherapy. Among these 19 patients, 9 (47.3\%) had teratoma and $3(15.8 \%)$ had live tumour cells. In group 2, $6(13.9 \%)$ of the 43 patients underwent RPLND. Two of these 6 patients had live tumour cells and teratoma simultaneously. In group 2, $10(23.2 \%)$ of the 43 patients underwent RPLND after chemotherapy. Five of these 10 patients (50\%) had teratoma, while 4 (40\%) had live tumour cells. Statistical analysis was not performed due to the small cohort of included patients that underwent RPLND.

The histopathological, demographic and clinical data for the 43 MGCT patients having a seminoma component are summarised in Table 4. The cases were followed 
Table 4. The histopathological, demographic and clinical data of patients in group 2

\begin{tabular}{|c|c|c|c|c|c|c|c|c|c|c|c|c|c|c|}
\hline Case & $\begin{array}{l}\text { Age, } \\
\text { years }\end{array}$ & $\begin{array}{l}\text { USG } \\
\text { tumour } \\
\text { size, } \\
\mathrm{mm}\end{array}$ & $\begin{array}{l}\text { Site of } \\
\text { tumour }\end{array}$ & LVI & $\begin{array}{l}\text { Pathological } \\
\text { T stage }\end{array}$ & $\begin{array}{l}\text { Seminoma } \\
\text { rate, \% }\end{array}$ & $\begin{array}{l}\text { Dominant } \\
\text { compo- } \\
\text { nent }\end{array}$ & $\begin{array}{l}\text { Tumour } \\
\text { markers }^{\mathrm{a}}\end{array}$ & $\begin{array}{l}\text { Metastasis of } \\
\text { retroperito- } \\
\text { neal lymph node } \\
\text { in } \mathrm{CT}^{\mathrm{a}}\end{array}$ & $\begin{array}{l}\text { Lung metastasis } \\
\text { in } \mathrm{CT}^{\mathrm{a}}\end{array}$ & $\begin{array}{l}\text { Primary } \\
\text { treatment }\end{array}$ & $\begin{array}{l}\text { Adjuvant } \\
\text { treatment }\end{array}$ & $\begin{array}{l}\text { Follow-up } \\
\text { period, } \\
\text { months }\end{array}$ & Outcome \\
\hline 1 & 24 & 50 & left & positive & pT3 & 90 & $\mathrm{~S}$ & normal & 40 & undetectable & $\mathrm{RO}+\mathrm{CHT}$ & RPLND & 63 & $\begin{array}{l}\text { alive and free } \\
\text { from disease }\end{array}$ \\
\hline 2 & 25 & 20 & right & negative & pT1 & 90 & $\mathrm{~S}$ & high & undetectable & undetectable & $\begin{array}{l}\text { RO + } \\
\text { RPLND }\end{array}$ & - & 55 & $\begin{array}{l}\text { alive and free } \\
\text { from disease }\end{array}$ \\
\hline 3 & 21 & 40 & left & negative & pT1 & 90 & $\mathrm{~S}$ & high & 10 & undetectable & $\mathrm{RO}+\mathrm{CHT}$ & - & 26 & $\begin{array}{l}\text { alive and free } \\
\text { from disease }\end{array}$ \\
\hline 4 & 21 & 28 & left & positive & pT2 & 70 & $\mathrm{~S}$ & high & 15 & 20 & $\mathrm{RO}+\mathrm{CHT}$ & - & 12 & $\begin{array}{l}\text { alive and free } \\
\text { from disease }\end{array}$ \\
\hline 5 & 21 & 25 & left & negative & pT1 & 70 & $\mathrm{~S}$ & normal & 25 & undetectable & $\mathrm{RO}+\mathrm{CHT}$ & - & 106 & $\begin{array}{l}\text { alive and free } \\
\text { from disease }\end{array}$ \\
\hline 6 & 21 & 12 & right & negative & pT1 & 65 & $\mathrm{~S}$ & normal & undetectable & undetectable & $\begin{array}{l}\text { RO + } \\
\text { RPLND }\end{array}$ & - & 23 & $\begin{array}{l}\text { alive and free } \\
\text { from disease }\end{array}$ \\
\hline 7 & 20 & 25 & right & negative & pT1 & 60 & $\mathrm{~S}$ & high & 20 & 10 & $\mathrm{RO}+\mathrm{CHT}$ & - & 12 & $\begin{array}{l}\text { alive and free } \\
\text { from disease }\end{array}$ \\
\hline 8 & 25 & 30 & right & negative & pT1 & 50 & S/EC & normal & undetectable & undetectable & $\mathrm{RO}+\mathrm{CHT}$ & - & 89 & $\begin{array}{l}\text { alive and free } \\
\text { from disease }\end{array}$ \\
\hline 9 & 21 & 31 & left & negative & pT1 & 50 & $\mathrm{~S}$ & normal & 25 & undetectable & $\mathrm{RO}+\mathrm{CHT}$ & - & 16 & $\begin{array}{l}\text { alive and free } \\
\text { from disease }\end{array}$ \\
\hline 10 & 29 & 55 & right & positive & pT3 & 40 & $\mathrm{EC}$ & normal & 100 & undetectable & $\begin{array}{l}\text { RO + } \\
\text { RPLND }\end{array}$ & $\mathrm{CHT}$ & 20 & $\begin{array}{l}\text { alive and free } \\
\text { from disease }\end{array}$ \\
\hline 11 & 27 & 27 & left & negative & pT1 & 35 & $\mathrm{~T}$ & normal & undetectable & undetectable & $\begin{array}{l}\text { RO + } \\
\text { RPLND }\end{array}$ & - & 22 & $\begin{array}{l}\text { alive and free } \\
\text { from disease }\end{array}$ \\
\hline 12 & 20 & 42 & right & positive & pT2 & 35 & $\mathrm{EC}$ & normal & undetectable & undetectable & surveillance & CHT & 32 & recurrence \\
\hline 13 & 25 & 60 & left & negative & pT1 & 30 & $\mathrm{~T}$ & high & 25 & undetectable & $\mathrm{RO}+\mathrm{CHT}$ & RPLND & 29 & $\begin{array}{l}\text { alive and free } \\
\text { from disease }\end{array}$ \\
\hline 14 & 27 & 55 & right & positive & pT2 & 30 & $\mathrm{EC}$ & high & 55 & 14 & $\mathrm{RO}+\mathrm{CHT}$ & - & 16 & $\begin{array}{l}\text { alive and free } \\
\text { from disease }\end{array}$ \\
\hline 15 & 31 & 43 & right & positive & pT2 & 30 & $\mathrm{EC}$ & high & 35 & undetectable & $\mathrm{RO}+\mathrm{CHT}$ & RPLND & 111 & $\begin{array}{l}\text { alive and free } \\
\text { from disease }\end{array}$ \\
\hline 16 & 22 & 26 & right & negative & pT1 & 30 & $\mathrm{~T}$ & normal & 20 & undetectable & $\mathrm{RO}+\mathrm{CHT}$ & - & 26 & $\begin{array}{l}\text { alive and free } \\
\text { from disease }\end{array}$ \\
\hline 17 & 22 & 35 & right & positive & pT3 & 30 & $\mathrm{EC}$ & normal & 40 & 20 & $\mathrm{RO}+\mathrm{CHT}$ & - & 16 & $\begin{array}{l}\text { exitus due to } \\
\text { testicular } \\
\text { cancer }\end{array}$ \\
\hline 18 & 22 & 37 & right & positive & pT2 & 20 & $\mathrm{EC}$ & high & 30 & undetectable & $\mathrm{RO}+\mathrm{CHT}$ & - & 109 & $\begin{array}{l}\text { alive and free } \\
\text { from disease }\end{array}$ \\
\hline 19 & 27 & 20 & right & positive & pT2 & 20 & $\mathrm{EC}$ & normal & 20 & 10 & $\mathrm{RO}+\mathrm{CHT}$ & - & 6 & $\begin{array}{l}\text { alive and free } \\
\text { from disease }\end{array}$ \\
\hline 20 & 41 & 30 & right & positive & pT1 & 20 & EC & normal & undetectable & $\begin{array}{l}\text { undetect- } \\
\text { able }\end{array}$ & surveillance & - & 18 & $\begin{array}{l}\text { alive and free } \\
\text { from disease }\end{array}$ \\
\hline 21 & 33 & 32 & left & negative & pT1 & 20 & $\mathrm{~T}$ & normal & undetectable & undetectable & $\mathrm{RO}+\mathrm{CHT}$ & - & 7 & $\begin{array}{l}\text { alive and free } \\
\text { from disease }\end{array}$ \\
\hline 22 & 28 & 31 & right & negative & pT1 & 20 & $\mathrm{Y}$ & high & 40 & undetectable & $\mathrm{RO}+\mathrm{CHT}$ & - & 17 & $\begin{array}{l}\text { alive and free } \\
\text { from disease }\end{array}$ \\
\hline 23 & 21 & 25 & left & positive & pT2 & 15 & EC & normal & 13 & undetectable & $\mathrm{RO}+\mathrm{CHT}$ & RPLND & 21 & $\begin{array}{l}\text { alive and free } \\
\text { from disease }\end{array}$ \\
\hline 24 & 24 & 40 & right & negative & pT1 & 15 & $\mathrm{Y}$ & normal & 15 & undetectable & $\mathrm{RO}+\mathrm{CHT}$ & RPLND & 49 & $\begin{array}{l}\text { alive and free } \\
\text { from disease }\end{array}$ \\
\hline 25 & 34 & 32 & right & positive & pT2 & 15 & $\mathrm{EC}$ & high & 18 & undetectable & $\mathrm{RO}+\mathrm{CHT}$ & RPLND & 52 & recurrence \\
\hline 26 & 33 & 24 & right & positive & Pt3 & 10 & $\mathrm{EC}$ & high & 13 & undetectable & $\mathrm{RO}+\mathrm{CHT}$ & RPLND & 68 & $\begin{array}{l}\text { metastasis to } \\
\text { lung }\end{array}$ \\
\hline 27 & 25 & 40 & left & negative & pT1 & 10 & $\mathrm{EC} / \mathrm{T}$ & high & 30 & undetectable & $\mathrm{RO}+\mathrm{CHT}$ & RPLND & 41 & $\begin{array}{l}\text { alive and free } \\
\text { from disease }\end{array}$ \\
\hline 28 & 20 & 33 & left & negative & pT1 & 10 & $\mathrm{~T}$ & normal & 20 & undetectable & $\mathrm{RO}+\mathrm{CHT}$ & RPLND & 85 & $\begin{array}{l}\text { alive and free } \\
\text { from disease }\end{array}$ \\
\hline
\end{tabular}


Table 4 (continued)

\begin{tabular}{|c|c|c|c|c|c|c|c|c|c|c|c|c|c|c|}
\hline Case & $\begin{array}{l}\text { Age, } \\
\text { years }\end{array}$ & $\begin{array}{l}\text { USG } \\
\text { tumour } \\
\text { size, } \\
\mathrm{mm}\end{array}$ & $\begin{array}{l}\text { Site of } \\
\text { tumour }\end{array}$ & LVI & $\begin{array}{l}\text { Pathological } \\
\text { T stage }\end{array}$ & $\begin{array}{l}\text { Seminoma } \\
\text { rate, } \%\end{array}$ & $\begin{array}{l}\text { Dominant } \\
\text { compo- } \\
\text { nent }\end{array}$ & $\begin{array}{l}\text { Tumour } \\
\text { markers }^{\text {a }}\end{array}$ & $\begin{array}{l}\text { Metastasis of } \\
\text { retroperito- } \\
\text { neal lymph node } \\
\text { in } \mathrm{CT}^{\mathrm{a}}\end{array}$ & $\begin{array}{l}\text { Lung metastasis } \\
\text { in CT }\end{array}$ & $\begin{array}{l}\text { Primary } \\
\text { treatment }\end{array}$ & $\begin{array}{l}\text { Adjuvant } \\
\text { treatment }\end{array}$ & $\begin{array}{l}\text { Follow-up } \\
\text { period, } \\
\text { months }\end{array}$ & Outcome \\
\hline 29 & 20 & 29 & left & negative & pT1 & 10 & EC & normal & 20 & undetectable & $\mathrm{RO}+\mathrm{CHT}$ & - & 12 & $\begin{array}{l}\text { alive and free } \\
\text { from disease }\end{array}$ \\
\hline 30 & 24 & 25 & left & negative & pT1 & 10 & $\mathrm{EC}$ & normal & undetectable & undetectable & surveillance & - & 13 & $\begin{array}{l}\text { alive and free } \\
\text { from disease }\end{array}$ \\
\hline 31 & 21 & 65 & left & positive & pT2 & 10 & $\mathrm{EC}$ & high & 10 & 10 & surveillance & - & 12 & $\begin{array}{l}\text { alive and free } \\
\text { from disease }\end{array}$ \\
\hline 32 & 31 & 57 & left & negative & pT1 & 10 & $\mathrm{Y}$ & high & undetectable & undetectable & $\mathrm{RO}+\mathrm{CHT}$ & - & 18 & $\begin{array}{l}\text { alive and free } \\
\text { from disease }\end{array}$ \\
\hline 33 & 21 & 100 & right & positive & pT3 & 9 & $\mathrm{EC}$ & high & 120 & 15 & $\mathrm{RO}+\mathrm{CHT}$ & RPLND & 15 & $\begin{array}{l}\text { alive and free } \\
\text { from disease }\end{array}$ \\
\hline 34 & 25 & 34 & right & negative & pT1 & 5 & $\mathrm{Y}$ & normal & undetectable & undetectable & surveillance & - & 18 & $\begin{array}{l}\text { alive and free } \\
\text { from disease }\end{array}$ \\
\hline 35 & 21 & 30 & right & negative & pTl & 5 & $\mathrm{~T}$ & normal & undetectable & undetectable & surveillance & - & 25 & recurrence \\
\hline 36 & 32 & 56 & right & negative & pT1 & 5 & $\mathrm{~T}$ & normal & undetectable & undetectable & surveillance & - & 8 & $\begin{array}{l}\text { alive and free } \\
\text { from disease }\end{array}$ \\
\hline 37 & 27 & 20 & right & positive & pT2 & 5 & $\mathrm{~T}$ & high & 10 & 5 & $\mathrm{RO}+\mathrm{CHT}$ & - & 25 & $\begin{array}{l}\text { alive and free } \\
\text { from disease }\end{array}$ \\
\hline 38 & 33 & 41 & left & negative & pT1 & 5 & EC & normal & 10 & undetectable & $\begin{array}{l}\mathrm{RO}+ \\
\text { RPLND }\end{array}$ & - & 36 & $\begin{array}{l}\text { alive and free } \\
\text { from disease }\end{array}$ \\
\hline 39 & 20 & 27 & left & negative & pT1 & 5 & EC & normal & undetectable & undetectable & surveillance & - & 13 & $\begin{array}{l}\text { alive and free } \\
\text { from disease }\end{array}$ \\
\hline 40 & 27 & 30 & left & negative & pT1 & 5 & EC & normal & undetectable & undetectable & surveillance & - & 12 & $\begin{array}{l}\text { alive and free } \\
\text { from disease }\end{array}$ \\
\hline 41 & 22 & 35 & right & positive & pT2 & 4 & $\mathrm{Y}$ & normal & 10 & 5 & $\mathrm{RO}+\mathrm{CHT}$ & - & 71 & $\begin{array}{l}\text { metastasis to } \\
\text { brain }\end{array}$ \\
\hline 42 & 31 & 45 & right & negative & pTl & 2 & $\mathrm{~T}$ & high & undetectable & undetectable & $\begin{array}{l}\mathrm{RO}+ \\
\text { RPLND }\end{array}$ & - & 101 & $\begin{array}{l}\text { alive and free } \\
\text { from disease }\end{array}$ \\
\hline 43 & 28 & 75 & right & negative & pT1 & 1 & $\mathrm{~T}$ & normal & undetectable & undetectable & surveillance & - & 30 & $\begin{array}{l}\text { alive and free } \\
\text { from disease }\end{array}$ \\
\hline
\end{tabular}

USG, ultrasonography; EC, embryonal carcinoma; T, teratoma; Y, yolk sac tumour; S, seminoma; CT, computed tomography; RO, radical orchiectomy; CHT, chemotherapy; RPLND, retroperitoneal lymph node dissection; LVI, lympho-vascular invasion. ${ }^{\text {a Postoperatively. }}$

up for an average period of 36.2 months, and 37 of these cases did not exhibit recurrence during this follow-up period. During the follow-up period, 3 patients presented late retroperitoneal recurrence, and 1 patient had late brain metastasis. During the follow-up period, 1 other patient also passed away because of testicular cancer.

\section{Discussion}

Testicular tumours are a heterogeneous group of neoplasms that present different histopathologies and variable clinical courses and prognoses. Tumours that originate from the germ cells account for nearly 95\% of all testicular cancers [4]. According to the available literature [5], MGCTs are the second most common testicular germ cell tumours after seminomas and account for 40$45 \%$ of all the primary testicular GCTs. The reason why
MGCTs are this common may be linked to the fact that germ cells in the testicles are totipotent and undergo trophoblast or somatic differentiation. In the primary tumours or those that are metastatic, potent types of NSGCTs can transform into other NSGCT types [3]. The strong similarity between seminoma and intratubular germ cell neoplasia supports the hypothesis that seminoma is a precursor of other GCTs. According to this hypothesis, seminoma can differentiate and transform embryonal carcinoma and yolk sac tumours into MGCTs [6]. The same also applies to embryonal carcinomas, which, owing to their pluripotent nature, can transform into other NSGCTs and MGCTs such as teratomas, yolk sac tumours and choriocarcinomas through somatic differentiation [7]. Although the presence of seminomas within MGCTs is not generally considered as a negative prognostic factor, there are, as of yet, no published/reported studies on this topic in the literature [8]. 
In a study conducted by Miyai et al. [9] in 2018, it was reported that MGCTs containing a seminoma component exhibited genetic variations that were different from the ones observed in pure seminomas. Loss of heterozygosity was reported to be more frequent in MGCTs that had a seminoma component, and part of this loss of heterozygosity was reported to be associated with a loss of protein expression (i.e., PTEN). In the same study, a high level of allele losses was observed on the specific chromosomal loci of MGCTs that contain a seminoma component (i.e., $6 \mathrm{p}$ and 10q). The conclusion from this study was that although the word "seminoma" may initially suggest a good prognosis, the involvement of the seminoma component in MGCTs is not a good prognostic factor. Furthermore, considering the hypothesis that seminoma component in MGCTs is a precursor of other GCTs, it can be presumed that because MGCTs with a seminoma component have a high number of genetic anomalies, they can progress with greater malignancy.

In the light of these findings, the presence of seminoma in MGCTs should be evaluated clinically. With regard to age of the patients diagnosed with a testicular tumour, a bell-shaped curve of 10 years has been reported between seminomas and NSGCTs [10]. There was no clear relationship between the presence of seminoma in MGCTs and the age at diagnosis of the patients with MGCT in the literature. Only 1 study in the literature that separately examined MGCTs depending on whether they contained a seminoma component was reported. According to this study, the mean age of patients with MGCTs that lacked a seminoma component was reported as 29 years, while the mean age of patients with MGCTs that had a seminoma component was reported as 25 years [11]. However, the said study performed no statistical analysis between these 2 groups. The reason why no statistical analysis was performed between the 2 groups could be associated with the relatively small number of patients included. The number of patients with MGCTs that lacked a seminoma component was 30 , while the number of patients with MGCTs that had a seminoma component was 19 . The same study also reported that compared with MGCTs, pure seminomas were observed in patients who were statistically significantly older (the mean age was 34 years for pure seminomas and 27 years for MGCTs) [10]. In our study, the mean age of group 1 at the time of orchiectomy was 24.2 years, while the mean age of group 2 was 25.4 years, and this difference was not found to be significant (Table 1). However, as the seminoma component in MGCTs increases, the age of diagnosis of the patients with MGCTs increases. In our study, while those with a seminoma content below $30 \%$ behaved like the classic MGCT cases, when the seminoma rate was higher than $30 \%$, it exhibited a non-seminomatous behaviour. It is also seen at an early age and is aggressive. These findings were found to be concordant with Albers et al.'s [6] theory. Some seminomas have an aggressive behaviour and ability for differentiation at the cellular level. This condition supports their conversion to MGCTs, which is more frequently detected at an early age.

LVI in the primary tumour has been shown to robustly identify a group at higher risk of relapse [12]. Some studies have suggested that pure embryonal histology has significance, although it is less strongly associated with relapse than LVI [13]. However, there is limited information in the literature regarding the impact of the presence of a seminoma component on the disease prognosis. As is the case with other germ cell tumours, the rate of tumour spread in the retroperitoneal space is $70-80 \%$ [8]. Scanning for metastases after orchiectomy in the present study revealed that the rates of clinically significant lymph node involvement and lung metastasis in the patient group with MGCTs having a seminoma component were higher than in group 1. However, these differences were not statistically significant. In the clinical staging of both groups, 16 (37.2\%) patients with MGCTs having a seminoma component were determined to be at stage I, while this figure was 35 (51.4\%) in the other group. It has been reported in large case series that $55 \%$ of MGCTs were stage I, although there is no detailed information regarding the presence or absence of the seminoma component in them [14]. However, in the group of patients we studied, advanced stage (stages II and III) was identified more frequently in MGCTs that contained a seminoma component than in MGCTs that lacked a seminoma component.

It is reported that in NSGCTs, pathology evaluations for RPLND performed following chemotherapy revealed live tumour cells at a rate of 6-10\%, mature teratomas at a rate of $50 \%$ and necrotic-fibrotic tissues at a rate of $40 \%$ [15]. Another aspect that needs to be emphasised in the present study is that the RPLND pathology evaluations performed primarily and following chemotherapy revealed higher rates of live tumour cells in the group of patients with MGCTs having a seminoma component.

The results of the present study show that MGCTs that include a seminoma component (especially 30\% and higher) have a more advanced disease stage, greater lymph node involvement and a higher tendency for lung metastasis. The reason why these results have not been statistically supported can be explained by the low number of patients. The prognostic impact of the MGCTs that 
have a seminoma component should be re-examined in other case series with larger patient populations. The risk classification of the International Germ Cell Cancer Collaborative Group may undergo changes following the new studies conducted in this field [16].

The limitations of the present study included the retrospective evaluation of the data in a single centre and the lack of large patient cohorts with MGCTs containing or lacking a seminoma component. The 5-year overall survival rates of the patients could not be obtained because the follow-up period was not long enough.

\section{Conclusion}

Although the word "seminoma" may initially be interpreted as an indication of good prognosis, the presence of a seminoma component in MGCTs may not necessarily prove to be a good prognostic factor. MGCTs that contain a seminoma component (especially $30 \%$ and higher) can have a higher tendency for occult metastatic disease. The prognostic impact of the seminoma component in MGCTs should be re-examined in case series involving a higher number of patients.

\section{Statement of Ethics}

All procedures performed in studies involving human participants were in accordance with the ethical standards of the institutional and/or national research committee and with the $1964 \mathrm{Hel}-$ sinki Declaration and its later amendments or comparable ethical standards. Informed consent was obtained from all individual participants included in the study.

\section{Disclosure Statement}

The authors declare that they have no conflicts of interest.

\section{Funding Sources}

This research did not receive any specific grant from funding agencies in the public, commercial, or not-for-profit sectors.

\section{Author Contributions}

S.A.: study concept and design, analysis of data, intellectual oversight, manuscript preparation and editing. C.E.: study design, provision of patients, manuscript review. H.H.T.: statistical analysis, data interpretation, manuscript review and editing. A.O.: lead statistical design, study design, provision of patients. O.Y.: administrative and logistical support, manuscript review and editing.

\section{References}

1 Gurney JK, Florio AA, Znaor A, Ferlay J, Laversanne M, Sarfati D, et al. International Trends in the Incidence of Testicular Cancer: Lessons from 35 Years and 41 Countries. Eur Urol. 2019 Nov;76(5):615-23.

2 Damjanov I, Hes O. The effects of chemotherapy on metastatic testicular germ cell tumors. Open Pathol J. 2009 Sept;3(2):45-52.

3 Ulbright TM. Germ cell tumors of the gonads: a selective review emphasizing problems in differential diagnosis, newly appreciated, and controversial issues. Mod Pathol. 2005 Feb; 18(S2 Suppl 2):S61-79.

4 Bahrami A, Ro JY, Ayala AG. An overview of testicular germ cell tumors. Arch Pathol Lab Med. 2007 Aug;131(8):1267-80.

5 Williamson SR, Delahunt B, Magi-Galluzzi C, Algaba F, Egevad L, Ulbright TM, et al.; Members of the ISUP Testicular Tumour Panel. The World Health Organization 2016 classification of testicular germ cell tumours: a review and update from the International Society of Urological Pathology Testis Consultation Panel. Histopathology. $2017 \mathrm{Feb}$;0(3):335-46.

6 Albers P, Göll A, Bierhoff E, Schoeneich G, Müller SC. Clinical course and histopathologic risk factor assessment in patients with bilateral testicular germ cell tumors. Urology. 1999 Oct;54(4):714-8.
7 Rajpert-De Meyts E. Developmental model for the pathogenesis of testicular carcinoma in situ: genetic and environmental aspects. Hum Reprod Update. 2006 May-Jun;12(3): $303-23$.

8 Laguna MP, Albers P, Albrecht W, Algaba F, Bokemeyer C, Boormans JL, et al. EAU Guidelines on Testicular Cancer. European Association of Urology; 2019.

9 Miyai K, Ito K, Nakanishi K, Tsuda H. Seminoma component of mixed testicular germ cell tumor shows a higher incidence of loss of heterozygosity than pure-type seminoma. Hum Pathol. 2019 Feb;84:71-80.

10 Moch H, Cubilla AL, Humphrey PA, Reuter VE, Ulbright TM. The 2016 WHO Classification of Tumours of the Urinary System and Male Genital Organs-Part A: Renal, Penile, and Testicular Tumours. Eur Urol. 2016 Jul; 70(1):93-105.

11 Lobo J, Costa AL, Vilela-Salgueiro B, Rodrigues $\hat{A}$, Guimarães $\mathrm{R}$, Cantante $\mathrm{M}$, et al. Testicular germ cell tumors: revisiting a series in light of the new WHO classification and AJCC staging systems, focusing on challenges for pathologists. Hum Pathol. 2018 Dec;82: $113-24$.

12 Kollmannsberger C, Tandstad T, Bedard PL, Cohn-Cedermark G, Chung PW, Jewett MA, et al. Patterns of relapse in patients with clinical stage I testicular cancer managed with active surveillance. J Clin Oncol. 2015 Jan;33(1): 51-7.

13 Leão R, Ahmad AE, Hamilton RJ. Testicular Cancer Biomarkers: A Role for Precision Medicine in Testicular Cancer. Clin Genitourin Cancer. 2019 Feb;17(1):e176-83.

14 Klepp O, Flodgren P, Maartman-Moe H, Lindholm CE, Unsgaard $\mathrm{B}$, Teigum $\mathrm{H}$, et al. Early clinical stages (CS1, CS1Mk+ and CS2A) of non-seminomatous testis cancer. Value of pre- and post-orchiectomy serum tumor marker information in prediction of retroperitoneal lymph node metastases. Swedish-Norwegian Testicular Cancer Project (SWENOTECA). Ann Oncol. 1990 Jul; 1(4):281-8.

15 Carver BS, Serio AM, Bajorin D, Motzer RJ, Stasi J, Bosl GJ, et al. Improved clinical outcome in recent years for men with metastatic nonseminomatous germ cell tumors. J Clin Oncol. 2007 Dec;25(35):56038.

16 Mead GM, Stenning SP. The International Germ Cell Consensus Classification: a new prognostic factor-based staging classification for metastatic germ cell tumours. Clin Oncol (R Coll Radiol). 1997;9(4):207-9. 